\title{
PARTICLE ACCELERATION AT COMETS
}

\author{
Tamas I. Gombosi \\ Space Physics Research Laboratory \\ Department of Atmospheric, Oceanic and Space sciences \\ The University of Michigan, Ann Arbor, MI 48109
}

\section{ABSTRACT}

This paper compares calculated and measured energy spectra of implanted $\mathrm{H}^{+}$and $\mathrm{O}^{+}$ions on the assumption that the pick-up geometry is quasi-parallel and about 1 \& of the waves generated by the cometary pickup process propagates backward (towards the comet). The model provides a good description of the implanted $\mathrm{O}^{+}$and $\mathrm{H}^{+}$energy distribution near the plckup energies.

\section{INTRODUCTION}

Instruments at comets Giacobini-zinner and Halley detected large fluxes of energetic particles ${ }^{1-4}$. A significant part of the observed energetic ion population was detected at energies considerably larger than the pickup energy indicating the presence of some kind of acceleration process acting on implanted ions. Velocity diffusion of lower energy implanted ions (near the pickup energy) has also been observed by the several instruments upstream of the comet Halley bow shock ${ }^{5,6}$.

The acceleration of the implanted ions in the cometary upstream region has also generated considerable theoretical interest. This problem was first examined just before the Giacobini-zinner encounter ${ }^{7}$. In a subsequent paper written shortly before the Halley encounters, Ip and Axford considered five potential mechanisms that can act to accelerate implanted ions ${ }^{8}$. They concluded that in cometary environments the second-order Fermi acceleration (slow velocity diffusion due to the interaction with propagating Alfven waves) was likely to play a dominant role in accelerating ions of cometary origin far upstream from the comets. Their conclusion was also endorsed by Gribov et al. ${ }^{9}$. Later Isenberg published an elegant analytic solution for a specific scenario, which took into account convection, adiabatic acceleration and velocity diffusion ${ }^{10}$.

Shortly after Isenberg's analytic solution Gombosi developed a self-consistent, three-fluid model of plasma transport and implanted ion acceleration in the unshocked solar wind ${ }^{11}$. The model described convection, adiabatic and diffusive velocity change, as well as mass addition and charge exchange losses. Later, this model was extended to include the effects of first-order Fermi acceleration ${ }^{12}$ and the predicted velocity distribution functions were compared with 


\section{Particle Acceleration at Comets}

observations ${ }^{13}$. In this model a second-order Fermi mechanism accelerates ions to moderate energies in the cometary upstream region and then in the foreshock region (where the solar wind slows down from its ambient speed to about 0.8 times its upstream value) the superthermal implanted ions are further energized by a diffusive-compressive shock acceleration process (first-order Fermi acceleration) ${ }^{12,13}$.

\section{MODEL}

A well developed cometary atmosphere extends to distances some six orders of magnitude larger than the size of the nucleus. The dominant neutral molecules in this extended exosphere are $\mathrm{H}_{2} \mathrm{O}, \mathrm{CO}_{2}, \mathrm{CO}$ and their daughter products. Most of these neutral particles move wh velocities of about $1 \mathrm{~km} / \mathrm{s}$ with respect to the cometary nucleus and with a velocity of about $-u$ ( $u=s o l a r$ wind velocity) with respect to the plasma flow. Pickup of cometary particles, ionized by photoionization, charge exchange or electron impact, is the main physical process whereby comets interact with the solar wind.

Freshly born ions are accelerated by the motional electric field of the high-speed solar wind flow. The ion trajectory is cycloidal, resulting from the superposition of gyration and $\mathbf{E} \times \mathbf{B}$ drift. The resulting velocityspace distribution is a ring-beam distribution, where the gyration speed of the ring is $v_{1}=u$ sin $\alpha$, (where $u$ is the bulk plasma speed and $\alpha$ is the angle between the solar wind velocity and magnetic field vectors) and the beam velocity (along the magnetic field line) is $v_{1}=u \cos \alpha$. The ring beam distribution has large velocity space gradients and it is unstable to the generation of low frequency transverse waves.

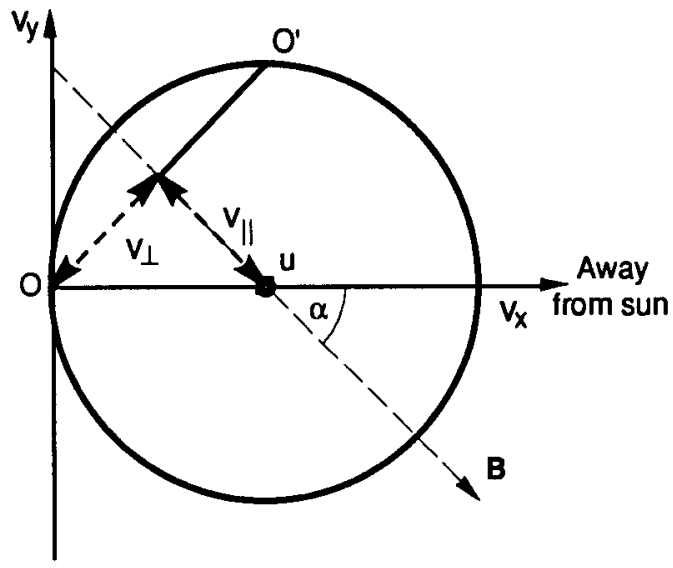

Fig. 1. Schematic representation of the implanted ion pickup geometry showing a velocity space diagram in the cometary frame of reference (the $v_{x}$ axis points from the sun towards the comet). The bulk velocity of the ambient plasma distribution is marked by u. The initial velocity of a newly born cometary is marked by 0 . The thin dashed line indicates the direction of the magnetic field. The initial velocityspace distribution is a ring-beam distribution. The projection of the pickup ring beam to the $\left(v_{x}, v_{y}\right)$ plane is denoted by the line $00^{y}$. 
In a first approximation the newly ionized pickup particles interact with the low frequency waves (sometimes called Alfven waves) without significantly changing their energy in the average wave frame. As a result of this process the pitch angles of the pickup-ring particles are scattered on the spherical velocity space shell of radius $u$ (see Fig. 1) around the local solar wind velocity. Observations indicate that this process does not lead to pitch-angle isotropy until very close to the cometary shock ${ }^{14,15}$.

- Our transport model is based on a series of recent papers discussing implanted ion acceleration processes in the upstream cometary region ${ }^{11-13}$. It takes into account advection, adiabatic acceleration, velocity diffusion (second order Fermi acceleration) and compressive-diffusive acceleration (first order Fermi acceleration) in a quasi-parallel geometry. Assuming that (i) the gyrophase distribution is totally random (this assumption, in effect means that diffusion across magnetic field lines is neglected), (ii) that the pitch-angle diffusion is a much faster process than energy diffusion, and (iii) the $\mathbf{u}$ and $\mathbf{B}$ vectors are parallel, one can derive the following Fokker-Planck equation for the pitch-angle averaged distribution function, $F(t, x, v)^{13}$ :

$$
\frac{\partial f}{\partial t}+u \frac{\partial f}{\partial z}-\frac{v}{3} \frac{d u}{d z} \frac{\partial f}{\partial v}=\frac{1}{v^{2}} \frac{\partial}{\partial v}\left(v^{2} D \frac{\partial f}{\partial v}\right)+\frac{\partial}{\partial z}\left(k \frac{\partial f}{\partial z}\right)+\frac{Q_{n} \delta(u-v)}{(4 \pi)^{2} \lambda_{n} v^{2} r^{2}} e^{-t / h_{o}}
$$

Here $x$ is distance along the flow line ( $x$ increases towards the comet), $r$ is cometocentric distance, $v$ is the implanted ion velocity in the plasma frame, $u$ is plasma bulk velocity, $D_{0}$ is the velocity diffusion coefficient, $\boldsymbol{K}$ is the field aligned spatial diffusion coefficient, $Q_{n}$ is the cometary gas production rate, and $\lambda_{n}$ is the ionization scale-length of a cometary neutral species. $F(t, x, v)$ is the pitch angle averaged phase-space density of implanted ions (separate equations are used to describe protons and water group ions) having random velocity magnitude $v$ at time $t$ and position $x$.

The first two terms on the left hand side of Equation (1) are the convective time derivative of the distribution function, while the third term describes energization due to adiabatic compression (in the cometary environment $d u / d x<0$, therefore this term results in energization of implanted particles). On the right hand side the first term describes energy diffusion in the plasma frame, the second term corresponds to spatial diffusion along magnetic field lines (in the present approximation this also corresponds to diffusion along flow lines), while the third term accounts for continuous production of cometary ions. The interplay between the adiabatic compression and spatial diffusion terms results in diffusive-compressive (first order Fermi) acceleration. 


\section{Particle Acceleration at Comets}

The spatial and velocity diffusion coefficients were obtained by using the quasilinear approximation. In this approximation the coefficients of spatial and velocity diffusions can be expressed in terms of the power spectrum of magnetic field fluctuations. However, one has to be careful, because different power spectra have to be used in the determination of these transport coefficients. When calculating $\kappa$, all magnetic field fluctuations have to be taken into account, regardless of their direction of propagation in the plasma frame of reference. When calculating the velocity diffusion coefficient, $D_{0}$, one has to recall that the wave field in the cometary environment is a superposition of fluctuations generated by the pickup process (these waves are predominantly low-frequency transverse waves propagating away from the comet along the magnetic field lines) and ambient waves in the solar wind (these waves move primarily away from the sun along the magnetic field lines). In the velocity diffusion coefficient an appropriately averaged magnetic field power spectrum has to be used to describe the effective power of randomly propagating (in both directions) magnetic field fluctuations. The details of this calculation can be found in our recent paper ${ }^{13}$.

\section{RESULTS AND DISCUSSION}

Implanted ion distributions were calculated using the model described in the previous section. The model solved the transport equation along flow lines (which in this particular model are identical to magnetic field lines). For each flow line the calculation started at $2.5 \times 10^{6} \mathrm{~km}$ upstream from the point where the flow Iine intersected the Glotto trajectory, and it extended to a distance of $1.5 \times 10^{6} \mathrm{~km}$ downstream from the intersection point. The $4 \times 10^{6} \mathrm{~km}$ distance (along the curved flow line) was divided into 200 spatial grid points resulting in a $2 \times 10^{4} \mathrm{~km}$ spatial step size. The velocity interval extended from 0 to $3500 \mathrm{~km} / \mathrm{s}$ (in the plasma frame) with a $25 \mathrm{~km} / \mathrm{s}$ step size. There were free escape boundaries at both ends of the flow line, and there was no flux through $\mathrm{v}=0$ and $\mathrm{v}=3500 \mathrm{~km} / \mathrm{s}$ (this velocity was high enough so that only an insignificant number of particles was accelerated to this value).

The model predictions were compared to observations made by the IMS HERS and JPA IIS instruments onboard the Giotto spacecraft. Fig. 2 shows a detailed comparison between observed and calculated energy spectra at a cometocentric distance of $\mathrm{r}=1.20 \times 10^{6} \mathrm{~km}$ along the inbound Giotto trajectory. The proton noise level is about $10^{-26.5} \mathrm{~s}^{3} / \mathrm{cm}^{6}$, therefore data points below this value should be ignored. The sharp rise of the proton phase space density function at low energies (below about $200 \mathrm{~km} / \mathrm{s}$ ) is due to the contribution of solar wind protons. The agreement between the calculated and observed spectra is very good. At the same time one should keep in mind 
that the plasma environment of comet Halley was a highly dynamic medium, with frequent changes of magnetic field configuration and flow patterns. Finally, the sensitivity of our results to some of the simplifications has to be discussed. One of the fundamental simplification of the model is the assumption of a parallel geometry, i.e. that the flow velocity and magnetic field vectors are parallel. Recent numerical simulations showed that for quasi-parallel geometries (when the angle, $\alpha$, between the flow velocity and the magnetic field vectors is less than about $60^{\circ}$ ) the backward propagating ratio of pickup generated waves is a couple of percent $^{17}$. This means that our velocity diffusion coefficient is applicable for situations with $\alpha<60^{\circ}$. In the case of quasiperpendicular geometry the effective wave power is much larger than in a quasi-parallel situation (because waves propagate in both directions with equal probability). The immediate consequence of the increased effective wave power is a large increase of the velocity diffusion coefficient. A temporary change from quasi-parallel to quasiperpendicular geometry might be the reason for the appearance of large energetic particle spikes observed in the cometary upstream region ${ }^{3,4}$.

\section{SUMMARY}

The results of model calculations describing transport and energization of cometary pickup ions were compared to observations made by the Giotto spacecraft. The model takes into account adiabatic compression, energy diffusion, field aligned spatial diffusion and mass addition along flow lines 


\section{Particle Acceleration at Comets}

of decelerating plasma flow lines. The model used the quasilinear approximation of the velocity and spatial diffusion coefficients. The generalized transport equation for the velocity-space solid-angle-averaged distribution function was solved for individual flow lines. The implanted ion spectra at the intersection points of the flow line with Giotto's trajectory were compared with spectra observed at that particular spatial location. The theoretical model provides a good description of the implanted proton and water group ion energy distribution near the pickup energy.

\section{ACKNOWLEDGEMENTS}

This work was supported by NASA grants NAGW-1366 and NAGW-2162. Acknowledgement is also made to the National Center for Atmospheric Research sponsored by NSF, for the computing time used in this research.

\section{REFERENCES}

1. R.J. Hynds, et al., Science, 232, 361 (1986).

2. F.M. Ipavich et al., Science, 232, 366 (1986).

3. K. Kecskeméty et al., J. Geophys. Res., 94, 185 (1989).

4. S. McKenna-Lawlor et al., Ann. Geophys., 7, 121 (1989).

5. A.J. Coates et al., J. Geophys. Res., 94, 9983 (1989).

6. M. Neugebauer et al., J. Geophys. Res., 24, 5227 (1989).

7. E. Amata and V. Formisano, Planet. Space Sci., 33, 1243 (1985).

8. W. - H Ip and W.I. Axford, Planet. Space Sci., 34, 1061 (1986).

9. B.E. Gribov et al., Astron. Astrophys., 187, 293 (1987).

10. P.A. Isenberg, J. Geophys. Res., 22, 8795 (1987).

11. T.I. Gombosi, J. Geophys. Res., 93, 35 (1988).

12. T.I. Gombosi et al., J. Geophys. Res., 94, 15011 (1989).

13. T.I. Gombosi et al., J. Geophys. Res., 96, 9467 (1991).

14. A.J. Coates et al., J. Geophys. Res., 95, 4343 (1990).

15. M. Neugebauer et al., J.Geophys. Res., 24, 1261 (1989).

16. J. Gaffey et al., J. Geophys. Res., 93, 5470 (1988).

17. R.H. Miller et al., J. Geophys. Res., 96, 9479 (1991). 